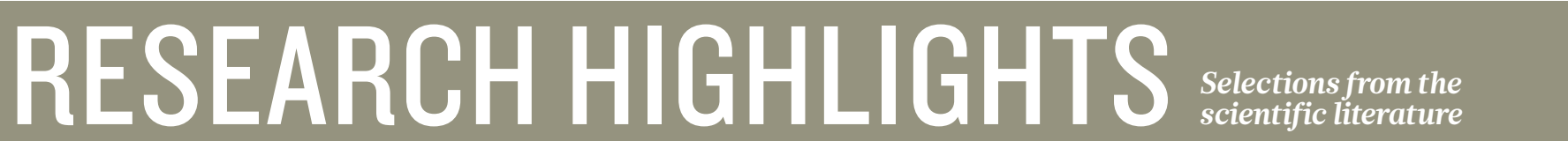

ASTROPHYSICS

\section{Cosmic boost reveals dim galaxy}

Astronomers have spied the faintest object ever seen in the early Universe.

Leopoldo Infante at the Pontifical Catholic University of Chile in Santiago and his team used NASA's Hubble and Spitzer space telescopes to study distant objects. They examined sections of the sky through a dense cluster of galaxies, which bends and magnifies incoming light, and found 22 faint galaxies. The oldest one was observed as it was 13.4 billion years ago, around 400 million years after the Big Bang.

The small, dim galaxy was named Tayna, meaning 'firstborn' in the Native South American language Aymara. It may be more representative of the first galaxies than other distant, brighter examples, say the authors.

Astrophys. J. 815, 18 (2015)

\section{GENOMICS}

\section{Missed mutations in cancer genomes}

A comparison of cancergenome sequences produced by 18 different research teams reveals that less than half of cancer-linked mutations were identified by all the groups. This suggests that differences in experimental procedures and analysis could reduce the accuracy of cancergenome sequencing, which is increasingly used in the clinic.

Ivo Gut at Spain's National Centre for Genomic Analysis in Barcelona, together with researchers in the International Cancer Genome Consortium, looked for genetic differences in cancerous and healthy tissue from the same person. They then compared these results

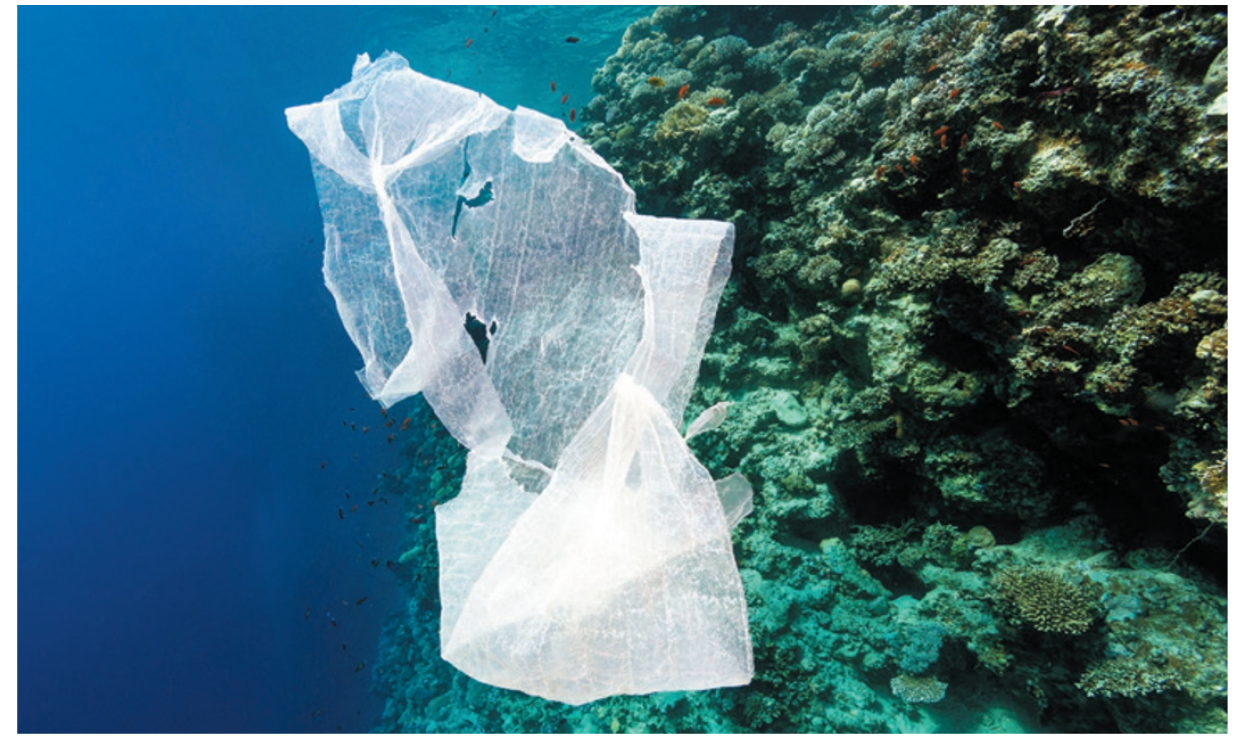

\section{ENVIRONMENTAL SCIENCE}

\section{Ocean plastic piling up fast}

Up to 240,000 tonnes of plastic particles are polluting the world's oceans - at least three times more than previous estimates.

Each year, 5 million to 13 million tonnes of plastic ends up in the sea, where it slowly degrades into microplastic particles that threaten marine ecosystems. Erik van Sebille at Imperial College London and his colleagues analysed 40 years of data on plastic collected from surface-trawling plankton nets - more information than in previous studies. By combining those data with sophisticated ocean-circulation models, they estimated that the oceans contain 93,000-236,000 tonnes of microplastic particles.

This represents just $1 \%$ of ocean plastic: the rest lies intact (pictured) on the sea floor or shore, or trapped in marine organisms, the authors suggest. Environ. Res. Lett. 10, 124006 (2015) with a benchmark that used ten times more sequencing data than usual. Out of more than 1,200 single-letter mutations, only $40 \%$ were identified by all 18 teams.

DNA preparation and other parameters can be optimized easily to improve sequencing accuracy, the authors say.

Nature Commun. 6, 10001 (2015)

\section{Rising sea levels alter Earth's spin}

Researchers have confirmed that rising sea levels caused by melting glaciers are slowing Earth's rotation.

As ice melts, it redistributes mass across the planet's surface, slightly changing the rate at which Earth spins. But a 2002 study could not explain the observed rotational changes on the basis of its assumptions about rising sea levels. Now Jerry Mitrovica of Harvard University in Cambridge, Massachusetts, and his colleagues say that they have resolved the problem. They used updated numbers for global sea-level rise, which are lower than those assumed in the 2002 study, and recalculated how the geographic poles have shifted over the past 3,000 years.

The work improves scientists' understanding of how Earth's rotation has changed in the past, and how rising sea levels might continue to alter it in the future.

Sci. Adv. 1, e1500679 (2015)

$$
\text { ASTRONOMY }
$$

\section{Galaxies caught in cosmic web}

Astronomers have discovered eight massive young galaxies within what might be a large web of dark matter.

Ordinary matter, including 